\title{
INTEGRAL DOMAINS WHOSE SIMPLE OVERRINGS ARE INTERSECTIONS OF LOCALIZATIONS
}

\author{
MARCO FONTANA, EVAN HOUSTON, AND THOMAS LUCAS
}

\begin{abstract}
Call a domain $R$ an sQQR-domain if each simple overring of $R$, i.e., each ring of the form $R[u]$ with $u$ in the quotient field of $R$, is an intersection of localizations of $R$. We characterize Prüfer domains as integrally closed sQQR-domains. In the presence of certain finiteness conditions, we show that the sQQR-property is very strong; for instance, a Mori sQQR-domain must be a Dedekind domain. We also show how to construct sQQR-domains which have (non-simple) overrings which are not intersections of localizations.
\end{abstract}

\section{INTRODUCTION}

Throughout this work, $R$ will denote an integral domain with quotient field $K$. The Kaplansky transform of an ideal $I$ of $R$ is denoted by $\Omega(I)$ and is defined by

$$
\Omega(I)=\left\{x \in K \mid \text { for each } a \in I \text { there is an integer } n(a) \geq 1 \text { such that } a^{n(a)} x \in R\right\} .
$$

(The notation $\Omega_{R}(I)$ will be used when the context involves more than one ring.) In [7] the first- and second-named authors studied domains $R$ each of whose overrings is a Kaplansky transform of an ideal $I$ of $R$. This work is in part a sequel to that paper. It turns out, however, that our investigations depend more heavily on the notions of unique minimal overrings and QQR-domains developed by Gilmer and Heinzer in [10. Recall that a domain $R$ is a $Q Q R$-domain if each overring of $R$ is an intersection of localizations of $R$. Davis [4] showed that a Prüfer domain must have the QQR-property and asked whether the converse is true. In their paper, Gilmer and Heinzer showed that the converse does not hold, and they explored in depth the relation between the Prüfer and QQR-properties.

We review basic definitions and prove basic facts in Section 10. In particular, we dub the domains of the title $s Q Q R$-domains, and we characterize Prüfer domains as being integrally closed sQQR-domains. The remainder of the paper is devoted to studying the non-integrally closed case. Since QQR-domains were characterized in [10, we are interested in sQQRdomains which are not QQR-domains. Much of Section 2 is concerned with showing that, in the presence of many frequently studied finiteness conditions, there are no such examples. For instance, we show that a Mori sQQR-domain is a Dedekind domain, that an sQQR-domain with Noetherian spectrum is a Prüfer domain, and that a semilocal sQQR-domain with treed spectrum is a QQR-domain. In Section 3 we do provide many (non-local) examples of

1991 Mathematics Subject Classification. 13A15, 13F05.

Key words and phrases. Prüfer domain.

All three authors acknowledge support from the Cultural Co-operation Agreement between Università degli Studi Roma Tre and The University of North Carolina at Charlotte; the first-named author was also partially supported by research grant MIUR 2001/2002 (Cofin 2000-MM01192794). 
sQQR-domains which are not QQR-domains. Finally, in the last section, we briefly discuss connections with seminormality.

\section{DEFINITIONS AND BASIC FACTS}

We begin by collecting some of the results we shall need. Recall that for an ideal $I$ of $R$, the $v$-closure of $I$ is defined by $I_{v}=\left(I^{-1}\right)^{-1}$ and the $t$-closure by $I_{t}=\bigcup J_{v}$, where the union is taken over all finitely generated subideals $J$ of $I$. We assume familiarity with these star operations.

Proposition 1.1. Let $I$ be an ideal of $R$. Then:

(1) $\Omega(I)=\bigcap\left\{R_{P} \mid P \in \operatorname{Spec}(R), I \nsubseteq P\right\}$.

(2) If I is finitely generated and $S$ is a multiplicatively closed subset of $R$, then $\Omega_{R}(I) R_{S}=$ $\Omega_{R_{S}}\left(I R_{S}\right)$.

(3) $\Omega(I)=\Omega(\operatorname{rad} I)$.

(4) If I has the property that for each $P \in \operatorname{Spec}(R)$ with $I \subseteq P$, we have $\Omega(I) \nsubseteq R_{P}$, then $\operatorname{rad} I$ is largest ideal $J$ for which $\Omega(I)=\Omega(J)$.

(5) $\Omega(I)=\Omega\left(I_{t}\right)$.

Proof. Statement (1) is proved in [1]. For statement (2), one sees easily that the Kaplansky transform coincides with the familiar Nagata transform for finitely generated ideals, and the Nagata transform is known to localize well. That $\Omega(I)=\Omega(\operatorname{rad} I)$ is [6. Lemma 3.1(c)]. For (4) suppose that $I \subseteq P$ and that $\Omega(J)=\Omega(I) \nsubseteq R_{P}$. By $(1), J \subseteq P$. Hence $J \subseteq \operatorname{rad} I$. Finally, (5) appears in [6. Proposition 3.4].

Notation. Let $R$ be a domain with quotient field $K$. If $U$ is a subset of $K$, we shall write $(R: U)$ for the fractional ideal $\{z \in K \mid z U \subseteq R\}$ and $\left(R:_{R} U\right)$ for the ideal $\{r \in R \mid r U \subseteq$ $R\}$.

Lemma 1.2. Let $R$ be a domain, and let $U$ be a subset of $K$. Then

(1) $R[U] \subseteq \Omega\left(R:_{R} U\right)$.

(2) If $U$ is finite, then $R[U]$ is the Kaplansky transform of an ideal of $R \Leftrightarrow R[U]=$ $\Omega\left(R:_{R} U\right)$.

Proof. Statement (1) follows easily from the definitions (with the convention that $\Omega(0)=K)$. Suppose that $U$ is finite and that $R[U]=\Omega(I)=\bigcap_{I \nsubseteq P} R_{P}$ for some ideal $I$ of $R$. Let $P$ be a prime ideal of $R$ with $I \nsubseteq P$. Then $U \subseteq R_{P}$, whence $\left(R:_{R} U\right) \nsubseteq P$. Thus $\Omega\left(R:_{R} U\right)=\bigcap_{\left(R:{ }_{R} U\right) \nsubseteq Q} R_{Q} \subseteq R_{P}$. Hence $\Omega\left(R:_{R} U\right) \subseteq \bigcap_{I \nsubseteq P} R_{P}=R[U]$. This proves $(2)$.

Lemma 1.3. Let $R$ be a domain with quotient field $K$. Then a finitely generated overring of $R$ is a Kaplansky transform of an ideal of $R$ if and only if it is an intersection of localizations of $R$.

Proof. Let $U$ be a finite subset of $K$, and suppose that there is a set of primes $\mathcal{P}$ in $R$ with $R[U]=\bigcap_{P \in \mathcal{P}} R_{P}$. For each $P \in \mathcal{P}$, we then have $\left(R:_{R} U\right) \nsubseteq P$. Hence

$$
R[U]=\bigcap_{P \in \mathcal{P}} R_{P} \supseteq \bigcap_{(R: R U) \nsubseteq Q} R_{Q}=\Omega\left(R:_{R} U\right) \supseteq R[U],
$$


the last inclusion following from Lemma 1.2. Thus $R[U]=\Omega\left(R:_{R} U\right)$. The converse holds in general (without the finiteness assumption) by Proposition [1.1](1).

Definition. We say that a domain $R$ is an $s Q Q R$-domain (respectively, $f Q Q R$-domain) if each simple overring (respectively, each finitely generated overring) of $R$ is an intersection of localizations of $R$.

Recall from [10] that a domain is a $Q Q R$-domain if each overring of $R$ is an intersection of localizations of $R$. In [7] the authors defined an $\Omega$-domain to be one for which each overring is a Kaplansky transform; hence a $\Omega$-domain is a QQR-domain. It was shown in [7] that the two properties are not the same. However, if one restricts to finitely generated overrings, then the properties are the same, as is shown by Lemma 1.3. Since [10] is a much older paper than [7, we have chosen to use the terminology "sQQR" (respectively, "fQQR") instead of "s $\Omega$ " (respectively, " $\mathrm{f} \Omega$ ").

In the integrally closed case, the sQQR- and fQQR-properties coincide (Proposition 2.1), but we have not been able to determine whether these properties coincide in general. We can easily see, however, that the sQQR- and $\Omega$-properties are distinct (even in the integrally closed case). This follows from the fact that a valuation domain is automatically a QQRdomain (and therefore an sQQR-domain), whereas a valuation domain need not be an $\Omega$ domain [7, Example 2.16].

In Section 3, we produce examples of sQQR-domains which are not QQR domains. However, we have not been able to produce local examples of sQQR-domains which are not QQR. Indeed, we devote much of the next section to showing that such examples cannot exist in the presence of certain finiteness assumptions.

\section{Finiteness Results}

We have the following result in the integrally closed case.

Proposition 2.1. Let $R$ be integrally closed. Then the following statements are equivalent.

(1) $R$ is an $s Q Q R$-domain.

(2) $R$ is an $f Q Q R$-domain.

(3) $R Q Q R$-domain.

(4) $R$ is a Prüfer domain.

Proof. Davis [4, p. 197] proved $(4) \Rightarrow(3)$. Hence it suffices to prove $(1) \Rightarrow(4)$. This is a familiar argument (cf. [8, proof of Theorem 26.2]). Let $u \in K$, and consider the simple overring $R\left[u^{2}\right]$. By hypothesis, this ring is an intersection of localizations of $R$ and is therefore integrally closed. Hence $u \in R\left[u^{2}\right]$, and so $u$ satisfies a polynomial $f$ over $R$ such that some coefficient of $f$ is a unit of $R$. It now follows from the $u, u^{-1}$-lemma (as in [8, Lemma 19.14]) that $R$ is a Prüfer domain.

Proposition 2.2. Let $R$ be an $s Q Q R$-domain which is not integrally closed, let $x \in \bar{R} \backslash R$, and let $\mathcal{P}$ denote the set of primes of $R$ which do not contain $\left(R:_{R} x\right)$. Then

(1) $\bigcup_{P \in \mathcal{P}} P$ is the set of nonunits of $R$, and

(2) $\mathcal{P}$ is infinite (so that $R$ has infinitely many primes). 
Proof. If $s \in R$ but $s \notin \bigcup_{P \in \mathcal{P}} P$, then $s$ is a unit of $\bigcap_{P \in \mathcal{P}} R_{P}=R[x]$ (the equality following from Lemma 1.2). Since $x$ is integral over $R$, this implies that $s$ is a unit of $R$. This proves (1). For (2), note that (1) implies that $\left(R:_{R} x\right) \subseteq \bigcup_{P \in \mathcal{P}} P$; prime avoidance then implies that $\mathcal{P}$ must be infinite.

This leads to our first restriction on (possible) local examples of sQQR-domains which are not QQR-domains.

Corollary 2.3. Let $(R, M)$ be a local sQQR-domain which is not integrally closed. Then

(1) $\operatorname{dim} R \geq 2$, and

(2) $M$ is not minimal over a principal ideal.

Proof. Statement (1) is clear from Proposition 2.2(2). Statement (2) follows from Proposition 2.2(1), upon observing that for a nonzero element $a \in M,\left(R:_{R} 1 / a\right)=(a)$.

Proposition 2.4. If $R_{M}$ is an $s Q Q R$-domain (respectively, $f Q Q R$-domain) for each maximal ideal $M$ of $R$, then $R$ is an $s Q Q R$-domain (respectively, fQQR-domain).

Proof. For $x \in K$, we have

$$
R[x]=\bigcap_{M \in \operatorname{Max}(R)}(R[x])_{R \backslash M}=\bigcap_{M \in \operatorname{Max}(R)} R_{M}[x] .
$$

For $M \in \operatorname{Max}(R)$, since $R_{M}$ is an sQQR-domain, $R_{M}[x]$ is an intersection of localizations of $R_{M}$ and therefore also of $R$. This takes care of the sQQR-property; the proof for the fQQR-property is similar.

Despite Proposition 2.4, neither the sQQR-property nor the fQQR-property is a local property. (We produce a specific example of this in Section 3.) In fact, in Proposition 2.12 below, we show that a domain which is locally a finite dimensional sQQR-domain is actually a Prüfer domain (and hence a QQR-domain).

We are able to localize the sQQR-property in some cases. The first such case is described in the following result; other cases will be examined in Remark 2.14 below.

Proposition 2.5. Let $R$ be a semilocal sQQR-domain with $\operatorname{Spec}(R)$ treed. Then $R_{M}$ is an $s Q Q R$-domain for each maximal ideal $M$ of $R$.

Proof. Fix a maximal ideal $M$. Let $x \in K \backslash M$, and let $\mathcal{P}$ (respectively, $\mathcal{Q}$ ) denote the set of primes which do not contain $\left(R:_{R} x\right)$ and which are contained in $M$ (respectively, are not contained in $M)$. Then we have $R[x]=\left(\bigcap_{P \in \mathcal{P}} R_{P}\right) \bigcap\left(\bigcap_{Q \in \mathcal{Q}} R_{Q}\right)$ by Proposition 1.1. Set $S=R \backslash M$. Then

$$
R_{M}[x]=R[x]_{S}=\left(\bigcap_{P \in \mathcal{P}} R_{P}\right) \bigcap_{S}\left(\bigcap_{Q \in \mathcal{Q}} R_{Q}\right)_{S}=\left(\bigcap_{P \in \mathcal{P}} R_{P}\right) \bigcap\left(\bigcap_{Q \in \mathcal{Q}} R_{Q}\right)_{S}
$$

We claim that $\left(\bigcap_{Q \in \mathcal{Q}} R_{Q}\right)_{S} \supseteq \bigcap_{P \in \mathcal{P}} R_{P}$. This will suffice to establish the result. To verify this, select a maximal ideal $N$ different from $M$. Since $\operatorname{Spec}(R)$ is treed, Zorn's lemma 
produces a unique prime ideal $N^{\prime}$ which is maximal with respect to being contained in $M \cap N$ (possibly, $N^{\prime}=0$ ). If $\left(R:_{R} x\right) \nsubseteq N^{\prime}$, then

$$
\left(\bigcap_{Q \in \mathcal{Q}, Q \subseteq N} R_{Q}\right)_{S} \supseteq\left(R_{N}\right)_{S}=R_{N^{\prime}} \supseteq \bigcap_{P \in \mathcal{P}} R_{P} .
$$

If $\left(R:_{R} x\right) \subseteq N^{\prime}$, then no $Q \in \mathcal{Q}$ satisfies $Q \subseteq N$, and the intersection "degenerates" to $K$. The claim now follows easily.

We require the concept of unique minimal overring: A proper overring $T$ of a domain $R$ is said to be the unique minimal overring of $R$ if each proper overring of $R$ is actually an overring of $T$. This concept was introduced in [10] as a tool for studying QQR-domains.

Proposition 2.6. Let $(R, M)$ be a local sQQR-domain, and suppose that $R \varsubsetneqq \Omega(M)$. Then

(1) $\Omega(M)$ is the unique minimal overring of $R$,

(2) $\Omega(M)$ is an $s Q Q R$-domain, and

(3) if $\Omega(M)=\bar{R}$, then $\bar{R}$ is a Prüfer domain.

Proof. (1) Let $T$ be a proper overring of $R$, and let $t \in T \backslash R$. Then $R[t]=\bigcap_{P \in \mathcal{P}} R_{P}$ for some set $\mathcal{P}$ of prime of $R$ with $M \notin \mathcal{P}$. Since $\Omega(M)=\bigcap_{Q \neq M} R_{Q}$, we have $\Omega(M) \subseteq R[t] \subseteq T$.

(2) Pick $x \in K$, and write $R[x]=\bigcap_{P \in \mathcal{P}} R_{P}$ for some set $\mathcal{P}$ of primes of $R$. Since $\Omega(M)$ is the unique minimal overring of $R$, this yields $R[x]=\Omega(M)[x]$; hence $\Omega(M)[x]$ is an intersection of localizations of $R$ and therefore also of $\Omega(M)$.

(3) An integrally closed sQQR-domain is a Prüfer domain by Proposition 2.1

Proposition 2.7. Let $(R, M)$ be a local sQQR-domain which is not integrally closed and for which $R \varsubsetneqq(R: M)$. Then $(R: M)=(M: M)=\Omega(M), \Omega(M)$ is the unique minimal overring of $R$, and (exactly) one of the following must occur:

(1) $(R: M)$ is local with maximal ideal $M$,

(2) $(R: M)$ is local with maximal ideal different from $M$, or

(3) $(R: M)$ has two maximal ideals $N_{1}, N_{2}$ with $N_{1} \cap N_{2}=M$.

Proof. By Corollary 2.3, $M$ is not principal. Since $M$ is maximal, we then have $(R: M)=$ $(M: M)$, and this is a proper overring of $R$. Since $(R: M) \subseteq \Omega(M)$, this implies that $(R: M)=\Omega(M)$ and that $\Omega(M)$ is the unique minimal overring of $R$ by Proposition 2.6. That this situation results in just the three cases mentioned follows from [10, Section 2].

The next result shows that in cases (1) and (3) above, $R$ is a QQR-domain. We recall that a prime ideal of a domain $D$ is unbranched if $P$ is the only $P$-primary ideal of $D$ and that in a Prüfer domain this is equivalent to having $P$ be the union of the prime ideals properly contained in $P$ [8, Theorem 23.3].

Proposition 2.8. Let $(R, M)$ be a local sQQR-domain which is not integrally closed and for which $R \varsubsetneqq(R: M)$. If $(R: M)$ is local with maximal ideal $M$ or if $(R: M)$ has two maximal ideals, then

(1) $(R: M)=\bar{R}$,

(2) $(R: M)$ is a Prüfer domain, and 
(3) $R$ is a QQR-domain.

Proof. We first deal with the case where $(R: M)$ has maximal ideal $M$. By [10, Proposition 2.6], $\bar{R}$ is a valuation domain with maximal ideal $M$. Hence for $u \in \bar{R}$, we have $u M \subseteq M$, and this implies that $(R: M)=\bar{R}$. Thus $\bar{R}=\Omega(M)=\bigcap_{P \neq M} R_{P}=\bigcap_{Q \in \mathcal{Q}} \bar{R}_{Q}$ for some set $\mathcal{Q}$ of primes of $\bar{R}$, with $Q \cap R \neq M$ for each $Q \in \mathcal{Q}$. Since $\bar{R}$ is a valuation domain, this implies that $M$ is unbranched in $\bar{R}$. By [10, Theorem 3.3], $R$ is a QQR-domain.

Now suppose that $(R: M)$ has two maximal ideals $N_{1}$ and $N_{2}$. In this case, $\bar{R}$ is a Prüfer domain, $M$ is an ideal of $\bar{R}$ and $N_{1} \cap N_{2}=M$ by [10, Proposition 2.5]. Hence for $u \in \bar{R}$, we have $M u \subseteq M \bar{R} \subseteq N_{1} \cap N_{2}=M$, and we have $u \in(R: M)$. Hence $\bar{R}=(R: M)$. We claim that each $N_{i}$ is unbranched. As before we have $\bar{R}=\bigcap_{P \neq M} R_{P}=\bigcap_{Q \in \mathcal{Q}} \bar{R}_{Q}$ for some set $\mathcal{Q}$ of primes in $\bar{R}$, with $Q \cap R \neq M$ for each $Q$. Hence for $Q \in \mathcal{Q}$, we must have $Q \neq N_{i}$ for $i=1,2$. If $N_{i}$ is branched, we can choose $t \in N_{i}$ but with $t$ in no other prime of $\bar{R}$. However, this yields $1 / t \in \bigcap_{Q \in \mathcal{Q}} \bar{R}_{Q}=\bar{R}$, a contradiction. Thus each $N_{i}$ is unbranched, and $R$ is a QQR-domain, again by [10, Theorem 3.3].

Recall that a pseudo-valuation domain is a local domain $(R, M)$ such that $(R: M)$ is a valuation domain with maximal ideal $M$. By Proposition 2.8, we have the following:

Corollary 2.9. Let $(R, M)$ be a PVD. Then $R$ is sQQR-domain $\Leftrightarrow R$ is a $Q Q R$-domain.

We are not able to rule out the possibility of an example as in case (2) of Proposition 2.7 The following result places some restrictions on such an example.

Proposition 2.10. Let $(R, M)$ be a local sQQR-domain which is not a $Q Q R$-domain. Suppose that $R$ is as in case (2) of Proposition 2.7. Set $T=(R: M)$, and let $N$ denote the maximal ideal of $T$. Then $T$ is non-integrally closed sQQR-domain, and $(T: N)=T=\Omega_{T}(N)$.

Proof. $T$ is an sQQR-domain by Proposition 2.6. Suppose, by way of contradiction, that $T$ is integrally closed. Then $T$ is a valuation domain by Proposition 2.1. In particular, we must have $T=\bar{R}$. The fact that $T=\bigcap_{P \neq M} R_{P}$ then implies that $T=\bigcap_{Q \in \mathcal{Q}} T_{Q}$ for some set $\mathcal{Q}$ of primes of $T$ with $N \notin \mathcal{Q}$. This, in turn, implies that $N$ is unbranched. However, $M$ is an ideal of $T$ and is therefore an $N$-primary ideal of $T$ different from $N$, a contradiction. Thus $T$ is not integrally closed. Now note that $(T: M)=\left(R: M^{2}\right) \subseteq \Omega(M)=T$, from which it follows immediately that $(T: N)=T$. Moreover, $\Omega(N)=\bigcap_{Q \nsupseteq N} T_{Q}=\bigcap_{Q \nsupseteq M T} T_{Q}=$ $\bigcap_{P \nsupseteq M} R_{P}=\Omega_{R}(M)=T$.

Corollary 2.11. If there is a local $s Q Q R$ domain $(R, M)$ which is not a $Q Q R$-domain, then there is such an example with $(R: M)=R=\Omega(M)$.

Proposition 2.12. Let $R$ be a domain such that $R_{M}$ is a finite dimensional sQQR-domain for each maximal ideal $M$ of $R$. Then $R$ is a Prüfer domain.

Proof. If $R$ is a local sQQR-domain of dimension 1 , then $R$ is a valuation domain by Corollary 2.3 and Proposition 2.1. Let $(R, M)$ be a local sQQR-domain of finite dimension greater than 1 . By induction, we may assume that $R_{P}$ is a valuation domain for each nonmaximal ideal $P$. If $R=\Omega(M)=\bigcap_{P \neq M} R_{P}$, then $R$ is an integrally closed sQQR-domain and is therefore a valuation domain by Proposition 2.1. Otherwise, $\Omega(M)$ is the unique minimal 
overring of $R$ by Proposition [2.6. and, again since each $R_{P}$ is integrally closed, we have $\Omega(M)=\bar{R}$. Hence $\bar{R}$ is a Prüfer domain by Proposition 2.6. Moreover, $\bar{R}$ has at most two maximal ideals [10, Corollary 2.2] and therefore $\bar{R}$, and hence also $R$, has only finitely many prime ideals. It now follows from Proposition 2.2 that $R=\bar{R}$. This takes care of local $R$. The general case follows easily.

We are now able to consider what happens when various finiteness conditions are imposed on an sQQR-domain. Recall that a domain $R$ is said to be $v$-coherent if for each finitely generated ideal $I$ of $R$, we have $I^{-1}=J_{v}$ for some finitely generated fractional ideal $J$ of $R$ and that $R$ is a finite conductor domain if each conductor ideal $\left(R:_{R} x\right)$, where $x \in K$, is finitely generated.

Proposition 2.13. If $R$ is an sQQR-domain which is locally finite dimensional, and if, furthermore, $R$ is v-coherent or a finite conductor domain, then $R$ is a Prüfer domain. In particular, a Noetherian sQQR-domain is a Dedekind domain.

Proof. Let $x \in K$. If $R$ is a finite conductor domain, then $\left(R:_{R} x\right)$ is finitely generated, and $R[x]$ is the Kaplansky transform of a finitely generated ideal. If $R$ is $v$-coherent, then $\left(R:_{R} x\right)=(R:(1, x))=I_{t}$ for some finitely generated ideal $I$. Hence by Proposition 1.1 and Lemma 1.2. we again have that $R[x]$ is the Kaplansky transform of a finitely generated ideal. It then follows from Proposition [1.1 that each localization of $R$ is also an sQQR-domain. The result now follows from Proposition 2.12

Recall that a domain $R$ is said to have Noetherian spectrum if it satisfies the ascending chain condition on radical ideals and to be a Mori domain if it satisfies the ascending chain condition on divisorial ideals.

Remark 2.14. From Proposition 2.4 and the proof of Proposition 2.13, we obtain that if $R$ is a v-coherent or a finite conductor domain, then $R$ is sQQR if and only if $R$ is locally $s Q Q R$. We shall see later (in the proof of Theorem 2.17) that the $s Q Q R$-property is also a local property for domains with Noetherian spectrum and for Mori domains.

Lemma 2.15. Let $(R, M)$ be a non-integrally closed local $s Q Q R$-domain which satisfies the ascending chain condition on radicals of conductor ideals. Then $\Omega(M)$ is the unique minimal overring of $R$.

Proof. Since $R$ is an sQQR-domain, for each $x \in K$ we have $R[x]=\Omega\left(R:_{R} x\right)=\Omega\left(\operatorname{rad}\left(R:_{R}\right.\right.$ $x)$ ) by Proposition 1.1 and Lemma 1.2. Moreover, the ideal $\left(R:_{R} x\right)$ satisfies the hypothesis of Proposition 1.1(4); hence for $x, y \in K$ we have $R[x] \varsubsetneqq R[y]$ if and only if $\operatorname{rad}\left(R:_{R} y\right) \varsubsetneqq$ $\operatorname{rad}\left(R:_{R} x\right)$. It then follows by our assumption that we may pick $u \in \bar{R} \backslash R$ such that $R[u]$ is a minimal extension of $R$ (i.e., there are no rings properly between $R$ and $R[u]$ ). By [10, Lemma 2.3], $M$ is the conductor of $R$ in $R[u]$, whence $R \varsubsetneqq(R: M) \subseteq \Omega(M)$. The lemma now follows from Proposition 2.6 .

It is known that in a Mori domain the radical of a divisorial ideal is again divisorial. Hence domains with Noetherian spectrum and Mori domains satisfy the ascending chain condition on radicals of conductor ideals.

Theorem 2.16. If $R$ is a Mori sQQR-domain, then $R$ is a Dedekind domain. 
Proof. We first handle the case where $R$ is actually assumed to be a Mori QQR-domain. In this case, since both properties localize, we may as well assume that $R$ is also local with maximal ideal $M$. If $R$ is integrally closed, then $R$ is a valuation domain, and it is well known that a Mori Prüfer domain is a Dedekind domain (see, e.g., the proof of [3. Proposition 2.6]). Hence we also assume that $R$ is not integrally closed. By [10, Theorem 3.3], $\bar{R}$ is a Prüfer domain, and the (at most two) maximal ideals of $\bar{R}$ are unbranched. However, if $Q$ is a nonzero, nonmaximal prime ideal of $\bar{R}$, then by [7, Lemma 3.4], $R_{Q \cap R}=\bar{R}_{Q}$, whence $R_{Q \cap R}$ is a Mori valuation domain. It is well known that this implies that $\bar{R}_{Q}$ is a rank one discrete valuation domain, whence $Q$ has height one. Thus the maximal ideals of $\bar{R}$ have finite height, which contradicts the fact that they are unbranched.

For the rest of the proof, we explicitly assume that $R$ is not a QQR-domain. Since for an ideal $I$ of a Mori domain, we have $I_{t}=I_{v}=J_{v}=J_{t}$ for some finitely generated ideal $J$ of $R$, we have $\Omega(I)=\Omega(J)$ by Proposition 1.1. so that the sQQR-property localizes. Hence we may assume that $R$ is a local Mori sQQR-domain with maximal ideal $M$. Of course, we may also assume that $R$ is not integrally closed. By Lemma 2.15] $\Omega(M)=(R: M)$ is the unique minimal overring of $R$. Hence $\Omega(M)$ is integral over $R$, and by [10, Lemma 2.3], $M$ is the conductor of $R$ in $\Omega(M)$. In particular, $M$ is divisorial. By Propositions [2.7, 2.8, and 2.10, $T=\Omega(M)$ is a local sQQR-domain which is not integrally closed, and the maximal ideal $N$ of $T$ satisfies $(T: N)=T$, so that $N$ is nondivisorial. Moreover, since $\Omega(M)=(R: M)$, $\Omega(M)$ is again a Mori domain by [13, I, Théorème 2]. However, as we just showed, this implies that the maximal ideal $N$ of $T$ is divisorial, a contradiction.

Theorem 2.17. Let $R$ be an sQQR-domain with Noetherian spectrum. Then $R$ is a Prüfer domain.

Proof. A ring with Noetherian spectrum has the property that each radical ideal is the radical of a finitely generated ideal. Hence each Kaplansky transform is the transform of a finitely generated ideal. It then follows from Proposition [1.1 that the sQQR-property localizes for domains with Noetherian spectrum. Hence we may assume that $R$ is a local sQQR-domain with maximal ideal $M$. We may also assume that $R$ is not integrally closed. If $R$ is a QQR-domain, then $M$ is unbranched by [10, Theorem 3.3]. However, $M$ is the radical of a finitely generated ideal, which contradicts that fact that $M$ is unbranched. (To see this, let $M=\operatorname{rad} J, J$ finitely generated. Since $M$ is unbranched, we must have $M=J$, i.e., $M$ is finitely generated. However, Nakayama's lemma then guarantees that $M \neq M^{2}$, and $M^{2}$ is then an $M$-primary ideal distinct from $M$, a contradiction.) Hence we may assume that $R$ is not a QQR-domain. By Lemma 2.15, $\Omega(M)$ is the unique minimal overring of $R$, and, as in the proof of Theorem 2.16, $M$ is the conductor of $R$ in $\Omega(M)$, so that $M$ is divisorial. Also as in the proof of Theorem 2.16, we obtain that $T=\Omega(M)$ is a local sQQR-domain with nondivisorial maximal ideal $N$. However, it is easy to see that the contraction map from $\operatorname{Spec}(T)$ to $\operatorname{Spec}(R)$ is a homeomorphism, from which it follows that $T$ also has Noetherian spectrum. But then the argument just given shows that $N$ is divisorial, a contradiction.

A one dimensional domain in which each nonzero element is contained in only finitely many primes has Noetherian spectrum. Hence we have the following result. 
Corollary 2.18. Let $R$ be a one-dimensional domain, and assume that each nonzero element of $R$ is contained in only finitely many primes. Then $R$ is an $s Q Q R$-domain $\Leftrightarrow R$ is a Prüfer domain.

Theorem 2.19. If $R$ is a semilocal $s Q Q R$-domain with treed spectrum, then $R$ is a $Q Q R$ domain.

Proof. By [10, Theorem 1.9], it suffices to prove that each localization of $R$ is a QQR-domain. Hence by Proposition 2.5, we may assume that $R$ is local with maximal ideal $M$. We may also assume that $R$ is not integrally closed. Let $x \in \bar{R} \backslash R$, and write $R[x]=\bigcap_{(R: R) \not P} R_{P}$. Since $x$ is integral over $R$, this intersection cannot contain $R_{Q}$ for any prime $Q \neq M$. Thus we must have $\operatorname{rad}\left(R:_{R} x\right)=M$. Hence $R[x]=\Omega(M)$. By Proposition 2.6, $\Omega(M)$ is the unique minimal overring of $R$, and, since $x$ was chosen arbitrarily, we have $\Omega(M)=\bar{R}$, so that $\bar{R}$ is a Prüfer domain. Note that in this situation $\bar{R}=\bigcap_{P \neq M} R_{P}$, and so $M$ must be the union of the chain of primes properly contained in $M$. We claim that each maximal ideal of $\bar{R}$ is unbranched. If not, let $N$ be a branched maximal ideal of $\bar{R}$. Then, since $\bar{R}$ is a Prüfer domain, $N=\operatorname{rad} \bar{R} u$ for some $u \in \bar{R}$ [8, Theorem 23.3]. By [10, Section 2], $u^{2} \in R$, whence $M=\operatorname{rad} R u^{2}$. However, this contradicts the fact that $M$ is the union of the chain of primes properly contained in $M$. Hence $R$ is a QQR-domain by [10, Theorem 3.3].

\section{EXAMPLES}

In this section, we construct examples of sQQR-domains which are not QQR-domains.

Lemma 3.1. Consider the following pullback diagram.

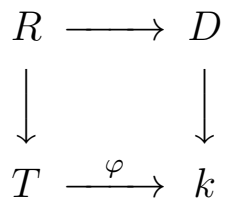

Here, $T$ is a domain, $k=T / M$ for some maximal ideal $M$ of $T, \varphi$ is the canonical homomorphism, and $D$ is a domain contained in $k$. Let $I$ be an ideal of $R$ which properly contains M. Then $\Omega_{R}(I)=\varphi^{-1}\left(\Omega_{D}(\varphi(I))\right)$.

Proof. Let $x \in \Omega_{R}(I)$. Note that since $I \supsetneqq M$ we have $x \in T$. Let $u=\varphi(a) \in \varphi(I)$, where $a \in I$. Then $x a^{n} \in R$ for some positive integer $n$. It follows that $\varphi(x) \varphi(a)^{n} \in D$. Thus $x \in \varphi^{-1}\left(\Omega_{D}(\varphi(I))\right)$. Now let $y \in \varphi^{-1}\left(\Omega_{D}(\varphi(I))\right)$, and let $b \in I$. We have $\varphi(y) \in \Omega_{D}(\varphi(I))$, so that $\varphi\left(y b^{m}\right)=\varphi(y) \varphi(b)^{m} \in D$ for some positive integer $m$; that is, $y b^{m} \in R$. It follows that $y \in \Omega_{R}(I)$.

Proposition 3.2. Consider the pullback diagram of Lemma [3.1, and assume that $T=V$ is a valuation domain and that $k$ is the quotient field of $D$. Then $R$ is an $s Q Q R$-domain (respectively, $f Q Q R$-domain) $\Leftrightarrow D$ is an sQQR-domain (respectively, $f Q Q R$-domain).

Proof. We give the details for the sQQR-case, the fQQR-case being similar. Assume that $R$ is an sQQR-domain. Let $u \in k$, and let $I=\varphi^{-1}\left(D:_{D} u\right)$. Pick $v \in V$ with $\varphi(v)=u$. Then $I=\left(R:_{R} v\right)$, and $R[v]=\Omega_{R}(I)$. Hence by Lemma 3.1] $D[u]=\varphi(R[v])=\varphi\left(\Omega_{R}(I)\right)=$ $\left.\Omega_{D}(\varphi(I))\right)=\Omega_{D}\left(D:_{D} u\right)$. Thus $D$ is an sQQR-domain. 
For the converse, let $x \in K$. If $x \in V$ we can use the same techniques to show that $R[x]=\Omega_{R}\left(R:_{R} x\right)$. If $x \notin V$, then $x^{-1} \in M \subseteq R$, and it is well known (and easy to show) that $R[x]=\Omega_{R}\left(x^{-1} R\right)$.

Proposition 3.3. Let $T$ be a Prüfer domain with the following properties:

(1) $T$ is one dimensional with $\operatorname{Max}(T)=\{M\} \cup \mathcal{P}$, with $\mathcal{P}$ infinite,

(2) Each element of $M$ is in $P$ for almost all $P \in \mathcal{P}$ (equivalently, each finitely generated ideal contained in $M$ is contained in $P$ for almost all $P \in \mathcal{P})$,

(3) Each element $a \in T \backslash M$ satisfies $a \notin P$ for infinitely many $P \in \mathcal{P}$, and

(4) $T / M$ admits a proper subfield $F$ such that there are no fields between $F$ and $T / M$.

Then $R$ is an $s Q Q R$-domain but is not a $Q Q R$-domain, where $R$ is defined by the following pullback diagram:

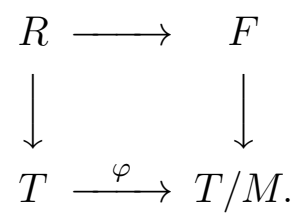

Moreover, $R_{M}$ is not an sQQR-domain.

Proof. Let $K$ denote the common quotient field of $R$ and $T$. Suppose that $\mathcal{Q}$ is an infinite subset of $\mathcal{P}$. Then for $u \in \bigcap_{Q \in \mathcal{Q}} T_{Q}$, we have that the finitely generated ideal $\left(T:_{T} u\right) \nsubseteq \mathbb{Q}$ for each $Q \in \mathcal{Q}$, whence by (2) $\left(T:_{T} u\right) \nsubseteq M$. Thus $u \in T_{M}$. That is, $\bigcap_{Q \in \mathcal{Q}} T_{Q} \subseteq T_{M}$ for each infinite subset $\mathcal{Q}$ of $\mathcal{P}$.

Now let $x \in K \backslash R$. We consider two cases.

Case 1. Suppose that $T[x] \subseteq T_{M}$. Then $\left(T:_{T} x\right) \nsubseteq M$, whence by $(3)\left(T:_{T} x\right) \nsubseteq P$ for infinitely many $P \in \mathcal{P}$. Thus $T[x]=\bigcap_{Q \in \mathcal{Q}} T_{Q}$ for some infinite subset $\mathcal{Q}$ of $\mathcal{P}$. Since $\left(T:_{T} x\right) \nsubseteq M$, we can choose $t \in T \backslash M$ with $t x \in T$. Now $t \notin M$ implies that $\varphi(t) \neq 0$, and we may find $t^{\prime} \in T$ with $\varphi\left(t t^{\prime}\right)=1$. Let $r=t t^{\prime}$. Then $r \in R \backslash M$, and $r x \in T$. If $r x \notin R$, then, since $r x \in T$ and $R \subseteq T$ is a minimal extension (there are no rings properly between $R$ and $T$ ), we have $T=R[r x] \subseteq R[x]$, whence $R[x]=T[x]=\bigcap_{Q \in \mathcal{Q}} T_{Q}=\bigcap_{Q \in \mathcal{Q}} R_{Q \cap R}$. Thus we may assume that $r x \in R$, so that $\left(R:_{R} x\right) \nsubseteq M$, and we have $R[x] \subseteq R_{M}$. We shall show, in fact, that $R[x]=T[x] \cap R_{M}$, which will complete the proof in this case. Note that $M[x]$ is a maximal ideal of $T[x]$, and it follows that $M[x]$ is also a maximal ideal of $R[x]$. Thus we have the following pullback diagram:

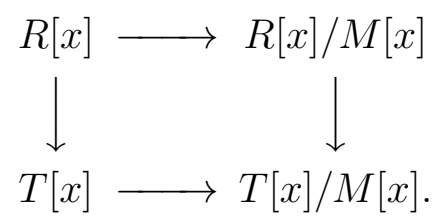

Moreover, $R / M \subseteq R[x] / M[x] \subseteq R_{M} / M R_{M}$, whence $R[x] / M[x]=F$. Similarly, $T[x] / M[x]=$ $T / M$. Hence $R[x] \subseteq T[x]$ is a minimal extension. However, we have $R[x] \subseteq T[x] \cap R_{M} \varsubsetneqq T[x]$, whence $R[x]=T[x] \cap R_{M}$, as desired.

Case 2. Suppose that $T[x] \nsubseteq T_{M}$. Then $\left(T:_{T} x\right) \subseteq M$, so that $\left(T:_{T} x\right) \subseteq P$ for almost all $P \in \mathcal{P}$ by (2). Hence $T[x]=T_{P_{1}} \cap \cdots \cap T_{P_{n}}$ for some finite subset $\left\{P_{1}, \ldots, P_{n}\right\}$ of $\mathcal{P}$. It follows that $M T[x]=T[x]$. However, $M T[x]=M[x]=M R[x] \subseteq R[x]$, so that 
$R[x]=T[x]=\bigcap_{j=1}^{n} T_{P_{j}}=\bigcap_{j=1}^{n} R_{P_{j} \cap R}$. This completes the proof that $R$ has the sQQRproperty.

Now by [10, Theorem 1.9] the QQR-property is a local property. Moreover, $M$ is branched ( $R$ is one dimensional), whence by [10, Theorem 3.3], $R_{M}$ does not have the QQR-property. Hence $R$ is not a QQR-domain. Finally, observe that, since $R_{M}$ is not integrally closed, Corollary 2.3 assures that $R_{M}$ is not an sQQR-domain.

There remains the construction of domains with the properties described in Proposition 3.3. Begin with an almost Dedekind domain $T_{0}$ which is not Dedekind (so that there are necessarily infinitely many maximal ideals), which has precisely one non-finitely generated maximal ideal $M$, and which satisfies property (2) of the proposition; 9, Example 2, pp. 338-339] is one such example. We claim that condition (3) of the proposition is then automatically satisfied. To see this pick $a \in T_{0} \backslash M$; then $(M, a)=T_{0}$, and we may choose $c \in M$ so that $(a, c)=T_{0}$. Since $c$ lies in almost all $P_{i}, a$ fails to be in almost all $P_{i}$. As for condition (4), if it is not already satisfied, replace $T_{0}$ by $T=T_{0}(X)\left(T_{0}(X)=T_{0}[X]_{S}\right.$, where $S$ is the multiplicatively closed subset of $T_{0}[X]$ consisting of all polynomials having unit content). By [8, Proposition 36.7], $T$ is again an almost Dedekind domain. Moreover, the maximal ideals of $T$ are just the extensions of the maximal ideals of $T_{0}$; hence $T$ has properties (1)-(3) above. The pertinent residue field is $T / M T(X)=\left(T_{0} / M\right)(X)$, which admits appropriate subfields $F$, e.g., $F=\left(T_{0} / M\right)\left(X^{2}\right)$.

Since valuation domains are (s)QQR-domains, Propositions 3.2 and 3.3 can be used to produce examples of arbitrary dimension of sQQR-domains which are not QQR-domains.

\section{Seminormality}

Recall that if $R$ is a domain with quotient field $K$, then $R$ is seminormal if whenever $x \in K$ with $x^{2}, x^{3} \in R$, we have $x \in R$. We show below that a QQR-domain is seminormal, but we have not been able to determine whether an sQQR-domain (or even an fQQRdomain) need be seminormal. The example of an sQQR-domain which is not a QQR-domain discussed at the end of Section 3 is seminormal (it is also an fQQR-domain), so a seminormal sQQR-domain need not be a QQR-domain, but we do not know whether a local seminormal sQQR-domain (or fQQR-domain) must be a QQR-domain.

Proposition 4.1. A QQR-domain is seminormal.

Proof. It suffices to establish the result locally. Hence we assume that $(R, M)$ is a local QQR-domain, and we may as well assume that $R$ is not Prüfer. By [10, Section 2 and Theorem 3.3] either $\bar{R}$ is a valuation domain with maximal ideal $M$ or $\bar{R}$ is a Prüfer domain with two maximal ideals $N_{1}, N_{2}$ such that $N_{1} \cap N_{2}=M$. In particular, $M$ is a radical ideal of $\bar{R}$. Now let $x \in K$ be such that $x^{2}, x^{3} \in R$. Then $x \in \bar{R}$. If $x^{2} \notin M$, then $x=x^{3} / x^{2} \in R$. If $x^{2} \in M$, then since $M$ is a radical ideal of $\bar{R}$, we have $x \in M \subseteq R$, as desired.

We close with a characterization of seminormal fQQR-domains.

Proposition 4.2. The following statements are equivalent for an $f Q Q R$-domain $R$.

(1) Each overring of $R$ is seminormal.

(2) Each integral finitely generated overring of $R$ is seminormal. 
(3) $R[x]=R\left[x^{2}, x^{3}\right]$ for each $x \in K$.

(4) $\operatorname{rad}\left(R:_{R} x\right)=\operatorname{rad}\left(R:_{R} x^{2}\right) \cap \operatorname{rad}\left(R:_{R} x^{3}\right)$ for each $x \in K$.

(5) $R$ is seminormal.

If $R$ satisfies any of these equivalent conditions, then $\bar{R}$ is a Prüfer domain.

Proof. The implications $(3) \Leftrightarrow(1) \Rightarrow(2) \Rightarrow(5)$ are straightforward. Let $x \in K$. Then, using Proposition [1.1, we have $R\left[x^{2}, x^{3}\right]=R[x] \Leftrightarrow \Omega\left(R:_{R}\left(x^{2}, x^{3}\right)\right)=\Omega\left(R:_{R} x\right)$. As in the proof of Lemma 2.15, this last equality holds $\Leftrightarrow \operatorname{rad}\left(R:_{R}\left(x^{2}, x^{3}\right)=\operatorname{rad}\left(R:_{R} x\right)\right.$. Since $\left(R:_{R}\left(x^{2}, x^{3}\right)\right)=\left(R:_{R} x^{2}\right) \cap\left(R:_{R} x^{3}\right)$, this proves (3) $\Leftrightarrow$ (4). Assume (5), and let $a \in\left(R:_{R}\left(x^{2}, x^{3}\right)\right)$. Then $a x^{2}, a x^{3} \in R$, whence $(a x)^{2},(a x)^{3} \in R$. Since $R$ is seminormal, $a x \in R$. Thus $\operatorname{rad}\left(R:_{R} x^{2}\right) \cap \operatorname{rad}\left(R:_{R} x^{3}\right) \subseteq \operatorname{rad}\left(R:_{R} x\right)$. The converse always holds. Hence $(5) \Rightarrow(4)$. Finally, the last statement holds by [1, Theorem 2.3].

\section{REFERENCES}

[1] D.F. Anderson, D. Dobbs, and J. Huckaba, On seminormal overrings, Comm. Algebra 10 (1982), 14211447 .

[2] J. Brewer and R. Gilmer, Integral domains whose overrings are ideal transforms, Math. Nach. 51 (1971), 255-267.

[3] P.-J. Cahen, S. Gabelli, and E. Houston, Mori domains of integer-valued polynomials, J. Pure Appl. Algebra 153 (2000), 1-15.

[4] E. Davis, Overrings of commutative rings, II, Trans. Amer. Math. Soc. 110 (1964), 196-212.

[5] D. Ferrand and J.-P. Olivier, Homomorphismes minimaux d'anneaux, J. Algebra 16 (1970), $461-471$.

[6] M. Fontana, Kaplansky ideal transform: a survey, Advances in commutative ring theory (Proceedings of third Fès (Morocco) conference on commutative algebra), Lecture Notes in Pure and Applied Mathematics 205, pp. 271-306, Dekker, New York, 1999.

[7] M. Fontana and E. Houston, On integral domains whose overrings are Kaplansky ideal transforms, J. Pure Appl. Algebra 163 (2001), 173-192.

[8] R. Gilmer, Multiplicative ideal theory, Dekker, New York, 1972.

[9] R. Gilmer, Overrings of Prüfer domains, J. Algebra 4 (1966), 331-340.

[10] R. Gilmer and W. Heinzer, Intersections of quotient rings of an integral domain, J. Math. Kyoto Univ. 7 (1967), 133-150.

[11] J. Hays, The S-transform and the ideal transform, J. Algebra 57 (1979), 223-229.

[12] J. Hedstrom and E. Houston, Pseudo-valuation domains, Pacific J. Math. 75 (1978), 137-147.

[13] N. Raillard (Dessagnes), Sur les anneaux de Mori, C.R. Acad. Sci. Paris Sér. A 280 (1975), 1571-1573.

Dipartimento di Matematica, Università degli Studi Roma Tre, Largo San L. Murialdo, 1, 00146 Roma, Italy

E-mail address: fontana@mat.uniroma3.it

(Evan Houston and Thomas Lucas) Department of Mathematics, University of North Carolina at Charlotte, Charlotte, NC 28223 U.S.A.

E-mail address, Evan Houston: eghousto@email.uncc.edu

E-mail address, Thomas Lucas: tglucas@email.uncc.edu 